\section{MMP12 makes the cut}

Matrix metalloproteinase 12 (MMP12), a protease involved in macrophage migration, could also have a crucial role in regulating the resolution of inflammation. The results of a new study indicate that MMP1 2 cleaves IFN $\gamma$, thereby rendering the cytokine unable to signal via its receptor.

In this study, the researchers investigated interactions between MMP12 and IFN $\gamma$ in vitro before using $\mathrm{Mmp} 12^{+/+}$and $\mathrm{Mmp} 12^{-/}$ mice with acute peritonitis to monitor the effects of these interactions in vivo.

"We found that MMP12 efficiently inactivates IFN $\gamma$ by two C-terminal cleavages that remove the IFN $\gamma$ receptor-binding site. These cleavages halt downstream signalling and classical-activation to M1 (IFN $\gamma$-activated) macrophages," explains corresponding author Christopher Overall. "Moreover, we found that M2 (IL-4-activated) macrophages express $>3$-fold more
MMP12 than the pro-inflammatory M1 macrophages. This suggests a new feedback inhibition mechanism executed by M2 cells that reduces the ratio of destructive $\mathrm{M} 1$ cells and so favours M2 cells and disease resolution."

Interestingly, the results from mice with peritonitis were mirrored in the MRL/lpr mouse model of systemic lupus erythematosus (SLE); IFN $\gamma$ signalling was prolonged in $M m p 12^{-/-}$mice compared with $M m p 12^{+/+}$mice and supported a sustained pro-inflammatory response.

Re-analysis of previously published transcriptome datasets revealed an inverse correlation between MMP12 mRNA and mRNA of IFN $\gamma$-response genes in patients with SLE that was not present in healthy individuals. Over time, this inverse correlation was associated with increased disease activity, but was reversed in patients who responded well to therapy.

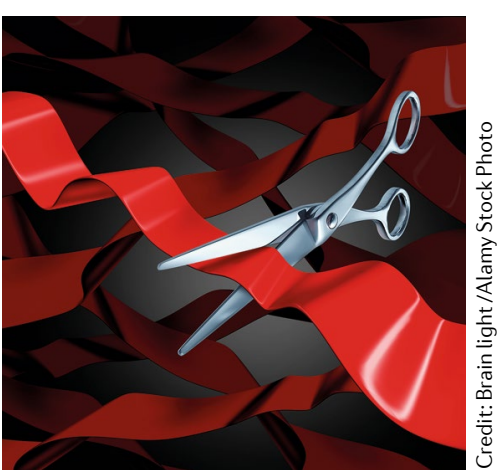

Furthermore, levels of MMP12cleaved IFN $\gamma$ were lower in tissue samples from the kidneys of patients with lupus nephritis than in those from healthy individuals.

"Macrophage activation patterns remain under-explored in autoimmune diseases, as does the post-translational regulation of cytokines. Genetic analyses alone will miss the important contribution made by proteolytic processing of cytokines," concludes Overall.

Joanna Collison

signal via its
receptor

\title{
VASCULITIS
}

\section{MIF in granulomatosis with polyangiitis}

Macrophage migration inhibitory factor (MIF) seems to have an important function in the pathogenesis of granulomatosis with polyangiitis (GPA) according to the results of a new study. In patients with GPA, vasculitis is associated with the formation of inflammatory granulomas. "There remains a lack of clear understanding regarding the formation of granulomas in these patients," explains corresponding author Antoine Sreih.

MIF is implicated in other granulomatous diseases, such as sarcoidosis and tuberculosis. "We used the comprehensive approach of combining a human genetic study with an experimental animal model to under stand the role of MIF in GPA," says Sreih.

Two MIF promoter polymorphisms have been identified: a -794 $\mathrm{CATT}_{6-8}$ microsatellite (rs5844572) and a -173 G/C SNP (rs755662). Thus, the researchers conducted a multicentre, cross-sectional genetic study of 501 patients with GPA and 576 healthy individuals. $60.2 \%$ of patients with GPA carried an increased number of -794 CATT $_{6-8}$ repeats in their genome, compared with $53.9 \%$ of healthy

$\uparrow$ Pulmonary granuloma formation

$\uparrow$ Neutrophil and macrophage chemokine production

$\uparrow$ Mortality

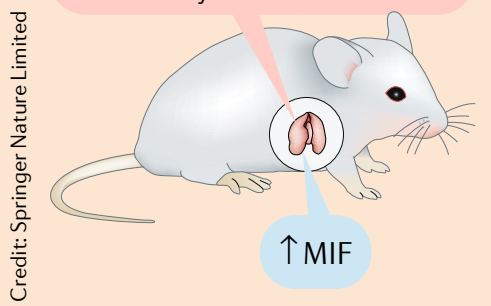

individuals. Patients with GPA also had higher plasma levels of MIF than their disease-free counterparts.

"The experimental model is a mouse model of granulomatous vasculitis induced by injection of Candida albicans $\beta$-glucan," explains Sreih. "To mimic the high-expression MIF polymorphisms in humans, we developed tissue-conditional transgenic mouse lines that overexpress MIF in lung epithelium."

Mice overexpressing lung epithelial MIF developed more pulmonary granulomas than wild-type mice; they also had increased levels of neutrophil and macrophage chemokines and increased mortality. Injection of an anti-MIF monoclonal antibody reduced lung disease and improved survival in these mice.

Notably, an anti-MIF receptor antibody is currently in phase II trials for the treatment of systemic lupus erythematosus, which, if successful, could have implications for the treatment of patients with GPA.

Isobel Leake

ORIGINAL ARTICLE Sreih, A. G. et al. Role of macrophage migration inhibitory factor in granulomatosis with polyangiitis. Arthritis Rheumatol.https://doi.org/10.1002/art.40655 (2018)
Injection of an anti-MIF monoclonal antibody ... improved survival in these mice 